Research Article

\title{
A Study to Assess the Effectiveness of Olive Oil Massage in Reducing Breast Engorgement and Pain among Postnatal Mothers with LSCS Admitted in Selected Hospital at Meerut
}

\author{
Priyanka Chaudhary', Tajnisha Banu' ${ }^{2}$, Ankur Farswal ${ }^{3}$
}

\author{
${ }^{1}$ Assistant Professor, M. Sc, Obstetrical and Gynaecological, Umalok College of Nursing, Meerut, Uttar Pradesh, India. \\ ${ }^{2}$ Reader, M. Sc, Obstetrical and Gynaecological, Nursing, PDM Subharti Nursing College, Subharti University, Meerut, Uttar \\ Pradesh, India. \\ ${ }^{3}$ Tutor, M.A (English), B. Sc, Meerut College, Uttar Pradesh, India. \\ DOI: https://doi.org/10.24321/2455.9318.201927
}

I $\mathbf{N}$ F O

Corresponding Author:

Priyanka Chaudhary, Obstetrical and Gynaecological, Umalok College of Nursing, Meerut, Uttar Pradesh, India.

E-mail Id:

priyankafarswal@gmail.com

Orcid Id:

https://orcid.org/0000-0002-5507-4280

How to cite this article:

Chaudhary P, Banu T, Farswal A. A Study to Assess the Effectiveness of Olive Oil Massage in Reducing Breast Engorgement and Pain among Postnatal Mothers with LSCS Admitted in Selected Hospital at Meerut. Int J Nurs Midwif Res 2019; 6(4): 13-21.

Date of Submission: 2019-12-14

Date of Acceptance: 2020-02-05

\section{$\begin{array}{llllllll}\mathbf{A} & \mathbf{B} & \mathbf{S} & \mathbf{T} & \mathbf{R} & \mathbf{A} & \mathbf{C} & \mathbf{T}\end{array}$}

Background: Breast feeding problems are encountered in up to $80 \%$ of mother and infant.

Objective: To assess the effectiveness of olive oil massage in reducing breast engorgement and pain among postnatal mothers with LSCS admitted in selected hospital at Meerut".

Materials and Methods: A quantitative approach with Quasi experimental, Non randomized control group design was selected as a research design. A total no of 60 postnatal mothers with LSCS who are suffering from breast engorgement and pain were purposively selected. 30 for experimental group and 30 for control group. Breast engorgement intensity was assessed by using six-point engorgement scale and breast pain intensity by a numerical pain rating scale. Olive oil massage was applied on an engorged and painful breast area for twice a day ,for duration of 10-15 min in experimental group. For control group no intervention was given. Posttest was assessed after $30 \mathrm{~min}$ of second intervention from both the group.

Result: The mean post-test score of experimental and control group for both right and left breast shows $79 \%$ reduction in engorgement score, this is statistically significant as evident from $p<0.001$ at 0.05 level and $71 \%$ reduction in pain score, this is statistically significant as evident from $p<0.001$ at 0.05 level. Posttest breast engorgement score of experimental groups with selected demographic variables shows significant association as evident from $P<0.05$. No association was found with posttest breast pain as $\mathrm{P}>0.05$.

Conclusion: Based on the findings the following conclusion can be drawn that application of olive oil massage is found effective in reducing breast engorgement and breast pain among postnatal mothers with LSCS.

Keywords: Olive Oil Massage, Breast Engorgement, Pain, Postnatal Mother with LSCS 


\section{Introduction}

Breastfeeding, problems are encountered in up to $80 \%$ of mother-infant. Breast engorgement occurs in $72 \%$ to $85 \%$ in post-natal mothers. Among every 10 mothers, 6 mothers suffer with breast engorgement.

Postnatal mothers suffer much distress after child birth during first week, due to breast engorgement and pain is most commonly associated with engorgement. In a hospital setting, engorgement is often seen in mothers who had operative or cesarean births. The incidence rate of breast engorgement all over the world is 1:8000 and in India it is 1:6500.

Breast engorgement can occur due to common reasons such as a suddenly increased milk production, delayed initiation of breastfeeding, infrequent feeds, ineffective suckling , sudden change in breast feeding routine and suddenly stopping breastfeeding. ${ }^{1}$

LSCS mothers need more care and attention than vaginal delivery mothers in a hospital setting, engorgement is seen often in mothers who have had operative or cesarean births so feedings are often delayed due to pain or reluctance to hold the baby in a position near the incision. Sometimes breastfeeding is delayed due to misinformation about medications the mother is receiving. ${ }^{2}$

A study was conducted to determine the barriers to timely initiation of breast feeding among mothers in WHO hospital. 500 consecutive health mother-infant both vaginally and by cesarean section were selected. Information was obtained using a structured questionnaire. Approximately $34 \%$ of the vaginal delivery mothers initiated breast feeding in early stage while no mother with cesarean section had early initiation of breast feeding. The mean time of breastfeeding initiation was $3.35 \pm 2.6$ hours. In mother who had vaginal delivery, $6.50 \pm 3.4$ hours and $5.9 \pm 1.9$ hours in those who had cesarean section with general or spinal anesthesia respectively. ${ }^{3}$

La Leche League, (2013). Massage is defined as the technique entails specific kneading, rubbing, and or squeezing strokes applied to the soft tissue of the breast to increase breast milk quantity and quality.

An experimental study was conducted on the effect of breast massage on the subjective discomfort of mother, the skin surface temperature changes in breast \& breast engorgement in Oita prefectural university of nursing in Japan. The subjects were 35 mothers \& midwife measured breast engorgement using visual analogue scale rating from $0-10$. The breast skin surface temperature measurement was assessed by infrared thermometer after 1 minutes, 3 minutes \& 5 minutes after breast massage. They concluded that breast massage is good for blood circulation and is considered to be an effective way to ease the discomfort of breast engorgement.

Olive oil contain MUFA (Mono Unsaturated Fatty Acids) enriches the body with good fat and lower the effect of bad cholesterol, Vit $\mathrm{E}$ strengthen immunity and Vit $\mathrm{K}$ aids in faster healing. It acts as sensual massage oil, it reduces bad cholesterol levels, makes your blood vessels more elastic, help fight breast cancer. Olive oil breast massage is a simple (and it has not any side effect) cost effective and easy method of treating breast engorgement and pain and also effective for sore nipples.

\section{Statement of the Problem}

A study to assess the effectiveness of olive oil massage in reducing breast engorgement and pain among postnatal mothers with LSCS admitted in selected hospital at Meerut".

\section{Objectives}

- To assess the level of breast engorgement and pain among postnatal mothers with LSCS in experimental and control group.

- To evaluate the effectiveness of olive oil massage in reducing breast engorgement and pain among postnatal mothers with LSCS in experimental group.

- To compare the effectiveness of olive oil massage in reducing breast engorgement and pain in experimental group with control group.

- To find the association between the posttest of breast engorgement and pain score of experimental groups with selected demographic variables.

\section{Materials and Method}

A quantitative approach with Quasi experimental, nonrandomized control group design was selected as a research design. Data were collected over the period of six weeks 10.2.2015 to 20.3.2015. Researcher selected a total number of 60 postnatal mothers with LSCS who are suffering from breast engorgement and pain was purposively selected from Dufferin district women hospital at Meerut. 30 for experimental group and 30 for control group. After obtaining ethical permission from institutional ethical committee of Subharti University, Meerut, a formal permission was obtained from Dufferin district women hospital, Meerut to conduct the final study. The tools were given for content validation to 9 experts from the field of nursing, clinical and naturopathy. Assessment of the tool reliability was done by conducting pilot study, in result the reliability coefficient was 0.50 and tools were found reliable. Pretest is assessed for breast engorgement intensity by using six-point engorgement scale and breast pain intensity by numerical pain rating scale. To apply Olive oil massage on engorged and painful breast for twice a day with six hours interval for duration 10-15 min for one day in Experimental group, in control group no intervention was given Posttest 
was assessed after 30 min of second intervention in both experimental and control group to reassess the level of breast engorgement and pain. Descriptive and inferential statistics were used for the data analysis.

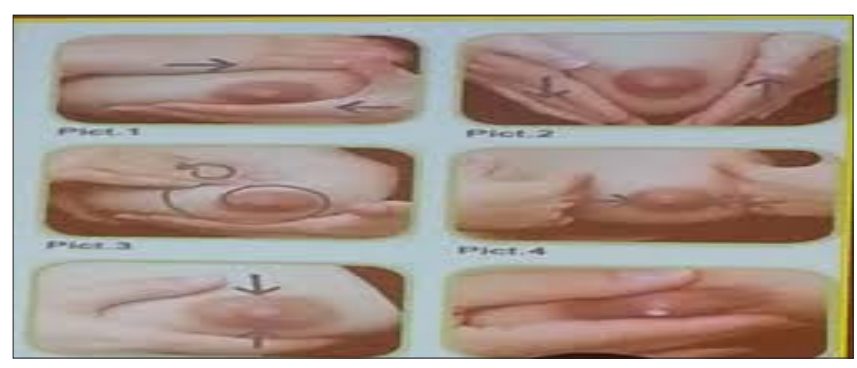

\section{Inclusion Criteria}

\section{Post Natal Mothers}

- Those who are willing to participate in the study.

- Those who are available at the time of data collection.

- Those who are complaining suffering from breast engorgement and pain.

- Those who are undergone LSCS.

\section{Exclusion Criteria}

\section{Postnatal Mothers}

- With Gestational diabetes Mellitus.

- Who are not willing to participate in the study.

- Suffering from Mastitis, breast abscess.

- With died baby.

\section{Description of Tool}

Section A: Socio-demographic Performa of postnatal mothers with LSCS

It consists of interview schedule seeking personal information like: age, education, occupation, parity, baby delivered gestational week, engorgement started day, baby status, previous knowledge, time interval of breast feeding, frequency of breast feeding, self-care method used and top feed/supplement given to neonate.

Section B: Six-point engorgement scale to assess the level of breast engorgement among postnatal mothers with LSCS

\begin{tabular}{|c|c|}
\hline Score & Description \\
\hline Score-1 & Soft no change in breast \\
\hline Score-2 & Slight change in breast \\
\hline Score-3 & Firm, non-tender breast \\
\hline Score-4 & Firm, beginning tenderness in breast \\
\hline Score-5 & Firm, tender \\
\hline Score-6 & Very firm, very tender \\
\hline
\end{tabular}

Section C: 11-point numerical pain rating scale to assess the level of pain among postnatal mothers with LSCS

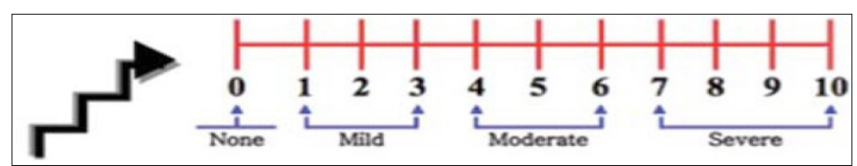

Figure 2.Numerical Rating Scale

The Numerical Rating Scale (NRS-11) is an 11-point scale for patient self-reporting of pain.

\begin{tabular}{|c|c|}
\hline Rating & Pain Level \\
\hline 0 & No Pain \\
\hline $1-3$ & Mild Pain \\
\hline $4-6$ & Moderate Pain \\
\hline $7-10$ & Severe Pain \\
\hline
\end{tabular}

Descriptive and inferential statistics were used for the data analysis.

\section{Section A}

Table I.Distribution of the Subjects according to demographic variables by frequency and percentage

\begin{tabular}{|c|c|c|c|c|}
\hline \multirow{2}{*}{ Sociodemographic variables } & \multicolumn{2}{|c|}{ Experimental group } & \multicolumn{2}{|c|}{ Control group } \\
\hline & Frequency (n) & Percentage (\%) & Frequency (n) & Percentage (\%) \\
\hline \multicolumn{5}{|l|}{ Age } \\
\hline $18-23$ years & 9 & 30 & 10 & 33.33 \\
\hline 24-29 years & 16 & 53.33 & 16 & 53.34 \\
\hline $30-35$ years & 5 & 16.67 & 4 & 13.33 \\
\hline Above 35 years & 0 & 0 & 0 & 0 \\
\hline \multicolumn{5}{|l|}{ Educational status } \\
\hline No formal education & 4 & 13.33 & 4 & 13.33 \\
\hline
\end{tabular}




\begin{tabular}{|c|c|c|c|c|}
\hline Primary school & 9 & 30 & 7 & 23.34 \\
\hline High school & 7 & 23.33 & 12 & 40 \\
\hline Higher secondary & 6 & 20 & 4 & 13.33 \\
\hline Graduate and above & 4 & 13.34 & 3 & 10 \\
\hline \multicolumn{5}{|l|}{ Occupation } \\
\hline Employed & 4 & 13 & 7 & 23 \\
\hline Self employed & 5 & 17 & 5 & 17 \\
\hline House wife & 21 & 70 & 18 & 60 \\
\hline \multicolumn{5}{|l|}{ Parity } \\
\hline Primipara & 18 & 60 & 16 & 53.34 \\
\hline Multipara & 10 & 33.34 & 11 & 36.66 \\
\hline Grand multipara & 2 & 6.66 & 3 & 10 \\
\hline \multicolumn{5}{|c|}{ Baby delivered at gestational week } \\
\hline$<36$ weeks (Preterm) & 6 & 20 & 8 & 26.66 \\
\hline At $37-40$ weeks (Term) & 20 & 66.66 & 18 & 60 \\
\hline$>40$ weeks (Post term) & 4 & 13.34 & 4 & 13.34 \\
\hline \multicolumn{5}{|c|}{ Engorgement started from the day of delivery } \\
\hline $2^{\text {nd }}-3^{\text {rd }}$ day & 17 & 57 & 15 & 50 \\
\hline $4^{\text {th }}-6^{\text {th }}$ day & 13 & 43 & 15 & 50 \\
\hline After $7^{\text {th }}$ day & 0 & 0 & 0 & 0 \\
\hline \multicolumn{5}{|l|}{ After delivery baby status } \\
\hline With mother & 19 & 63.34 & 20 & 66.66 \\
\hline In NICU & 11 & 36.66 & 10 & 33.34 \\
\hline \multicolumn{5}{|c|}{$\begin{array}{l}\text { Previous knowledge about breast feeding } \\
\text { practice }\end{array}$} \\
\hline Yes & 8 & 26.66 & 5 & 16.66 \\
\hline No & 22 & 73.34 & 25 & 83.34 \\
\hline \multicolumn{5}{|c|}{ Interval of breast feeding per day } \\
\hline Every 2 hourly & 9 & 30 & 5 & 16.66 \\
\hline Every 3 hourly & 6 & 20 & 9 & 30 \\
\hline Every 4 hourly & 8 & 26.66 & 12 & 40 \\
\hline Demand feed & 7 & 23.34 & 4 & 13.34 \\
\hline \multicolumn{5}{|c|}{ Frequency of breast feeding per day } \\
\hline$<6$ times & 14 & 46.66 & 13 & 43.34 \\
\hline 9-12 times & 16 & 53.34 & 17 & 56.66 \\
\hline$>12$ times & 0 & 0 & 0 & 0 \\
\hline \multicolumn{5}{|c|}{$\begin{array}{c}\text { Self-care method used for treatment of breast } \\
\text { engorgement }\end{array}$} \\
\hline Yes & 5 & 16.66 & 7 & 23.34 \\
\hline No & 25 & 83.34 & 23 & 76.66 \\
\hline \multicolumn{5}{|c|}{ Any top feed/ supplement being given to neonate } \\
\hline Yes & 4 & 13.34 & 6 & 20 \\
\hline No & 26 & 86.66 & 24 & 80 \\
\hline
\end{tabular}




\section{Section B}

Mean, S.D and mean percentage of pretest breast engorgement and pain Score of subjects among experimental and control group

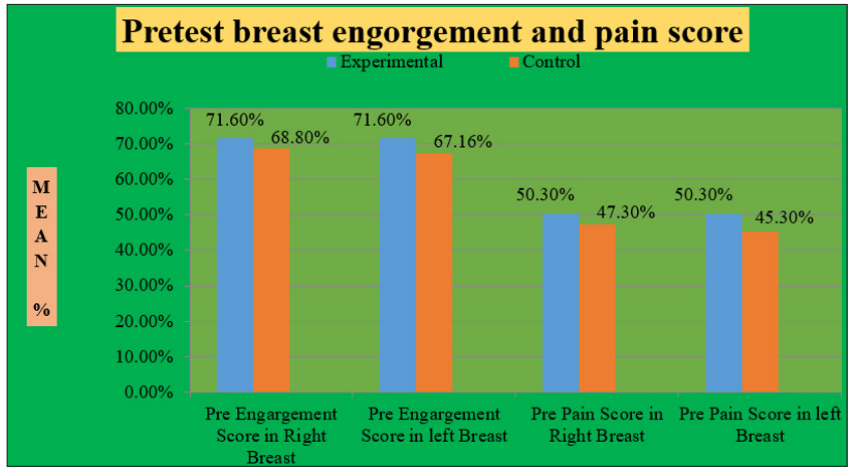

Figure 3.The multiple bar diagram showing mean percentage of pre test breast engorgement and pain Score in right and left breast in Experimental and Control group

The pretest mean engorgement score in right breast is slightly $3 \%$ higher in experimental group as compared to control group. A similar trend can be seen for the left breast $4.8 \%$ high as compared to control group. The pretest mean pain score in right breast is slightly higher $3 \%$ in experimental group as compared to control group. A similar trend can be seen for the left breast $5 \%$ high as compared to control group.
Mean, S.D and mean percentage of post-test engorgement and pain score of subjects among experimental and control group

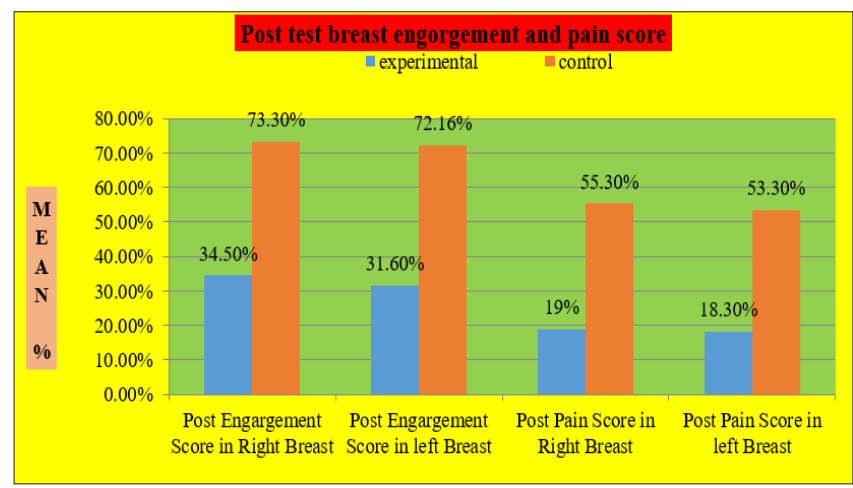

Figure 4.The multiple bar diagram showing mean percentage of Post-test breast engorgement and pain Score in right and left breast in Experimental and Control group

The mean posttest engorgement score for right breast was found $38.8 \%$ reduction in intensity of the right breast engorgement while for the left breast was found $40.56 \%$ reduction in the intensity of left breast engorgement. This was found statistically significant as evident from $\mathrm{p}<0.001$. The 'Mean' of the posttest pain score for the right breast was found $36.3 \%$ reduction in intensity of right breast pain and $35 \%$ reduction in the intensity of left breast pain. This found statistically significant as evident from $p<0.001$.

Table 2.Comparison of pretest and posttest breast engorgement score in experimental group with control group by 'unpaired t' test

$(\mathrm{N}=60)$

\begin{tabular}{|c|c|c|c|c|c|c|}
\hline \multirow{3}{*}{\multicolumn{2}{|c|}{ Parameter }} & \multicolumn{5}{|c|}{ Breast engorgement score } \\
\hline & & \multirow{2}{*}{$\begin{array}{c}\text { Experimental group } \\
\text { Mean } \pm S D\end{array}$} & \multirow{2}{*}{$\begin{array}{c}\text { Control group } \\
\text { Mean } \pm \text { SD }\end{array}$} & \multirow{2}{*}{$\begin{array}{l}\text { \% reduction/ } \\
\text { increment }\end{array}$} & \multirow{2}{*}{$\begin{array}{l}\text { Prob. Value's } \\
\text { of unpaired ' } t \text { ' test }\end{array}$} & \multirow{2}{*}{ p-value } \\
\hline & & & & & & \\
\hline \multirow{2}{*}{ Pre-test } & $\begin{array}{l}\text { Right } \\
\text { Breast }\end{array}$ & $4.30 \pm 1.02$ & $4.13 \pm 0.90$ & $3 \%$ & $\begin{array}{l}t=0.9686 \\
p=0.3407\end{array}$ & $\begin{array}{c}>0.001 \\
\text { (Non sig) }\end{array}$ \\
\hline & $\begin{array}{c}\text { Left } \\
\text { breast }\end{array}$ & $4.30 \pm 1.02$ & $4.03 \pm 0.99$ & $4.8 \%$ & $\begin{array}{l}t=1.0461 \\
p=0.3111\end{array}$ & $\begin{array}{c}>0.001 \\
\text { (Non sig) }\end{array}$ \\
\hline \multirow{2}{*}{ Post-test } & $\begin{array}{l}\text { Right } \\
\text { breast }\end{array}$ & $2.07 \pm 0.79$ & $4.40 \pm 1.04$ & $-39 \%$ & $\begin{array}{l}t=8.726 \\
P=0.000\end{array}$ & $\begin{array}{c}<0.001 \\
\text { (Sig) }\end{array}$ \\
\hline & $\begin{array}{c}\text { Left } \\
\text { breast }\end{array}$ & $1.90 \pm 0.80$ & $4.33 \pm 1.21$ & $-40.50 \%$ & $\begin{array}{l}P=0.000 \\
t=9.229\end{array}$ & $\begin{array}{c}<0.001 \\
\text { (Sig) }\end{array}$ \\
\hline
\end{tabular}

Table 3.Comparison of Pretest and Posttest breast pain score in experimental group with control group by 'unpaired t' test

\begin{tabular}{|c|c|c|c|c|c|c|}
\hline \multirow{2}{*}{ Parameter } & \multicolumn{5}{|c|}{ Breast pain score } \\
\cline { 2 - 7 } & Experimental group & Control group & $\begin{array}{c}\text { \% reduction/ } \\
\text { increment }\end{array}$ & $\begin{array}{c}\text { Prob. Value's } \\
\text { of unpaired 't' test }\end{array}$ & p-value \\
\cline { 2 - 8 } & Mean \pm SD & Mean \pm SD & & $\begin{array}{c}\mathrm{t}=0.748 \\
\mathrm{P}=0.779567\end{array}$ & $\begin{array}{c}>0.001 \\
\text { (NS) }\end{array}$ \\
\hline
\end{tabular}




\begin{tabular}{|c|c|c|c|c|c|c|}
\hline & $\begin{array}{c}\mathrm{LT} \\
\text { Breast }\end{array}$ & $5.03 \pm 1.71$ & $4.53 \pm 1.63$ & $5 \%$ & $\begin{array}{c}\mathrm{t}=1.627 \\
\mathrm{P}=0.251952\end{array}$ & $\begin{array}{c}>0.001 \\
\text { (NS) }\end{array}$ \\
\hline \multirow{2}{*}{ Post-test } & $\begin{array}{c}\mathrm{RT} \\
\text { Breast }\end{array}$ & $1.90 \pm 1.58$ & $5.53 \pm 1.74$ & $-36 \%$ & $\begin{array}{c}\mathrm{t}=8.465 \\
\mathrm{P}=0.001\end{array}$ & $\begin{array}{c}<0.001 \\
\text { (Sig) }\end{array}$ \\
\cline { 2 - 7 } & $\begin{array}{c}\mathrm{LT} \\
\text { Breast }\end{array}$ & $1.83 \pm 1.39$ & $5.33 \pm 1.94$ & $-35 \%$ & $\begin{array}{c}\mathrm{t}=8.045 \\
\mathrm{P}=0.001\end{array}$ & $\begin{array}{c}<0.001 \\
\text { (Sig) }\end{array}$ \\
\hline
\end{tabular}

Table 4.Presenting association between the posttest breast engorgement score of experimental group with selected demographic variables. By used ANOVA

$(\mathrm{N}=30)$

\begin{tabular}{|c|c|c|c|c|c|c|}
\hline \multirow{3}{*}{ Type of variables } & \multicolumn{6}{|c|}{ Mean breast engorgement score } \\
\hline & \multicolumn{3}{|c|}{ Rt breast } & \multicolumn{3}{|c|}{ Lt breast } \\
\hline & mean $\pm S D$ & $\mathbf{F}$ & p-value & mean $\pm S D$ & $\mathbf{F}$ & p-value \\
\hline $\begin{array}{c}\text { Age } \\
\text { 18-23 years }\end{array}$ & $1.67 \pm 0.50$ & \multirow{4}{*}{14.1} & \multirow{4}{*}{$\begin{array}{c}<0.001 \\
\text { (Sig) }\end{array}$} & $1.67 \pm 0.50$ & \multirow{4}{*}{8.57} & \multirow{4}{*}{$\begin{array}{c}0.001 \\
\text { (Sig) }\end{array}$} \\
\hline $24-29$ years & $2.56 \pm 0.62$ & & & $2.31 \pm 0.79$ & & \\
\hline 30-35 years & $1.20 \pm 0.44$ & & & $1.00 \pm .00$ & & \\
\hline Above 35 years & - & & & - & & \\
\hline \multicolumn{7}{|c|}{ Educational status } \\
\hline No formal Education & $2.50 \pm 0.57$ & \multirow{5}{*}{8.92} & \multirow{5}{*}{$\begin{array}{c}<0.001 \\
(\mathrm{Sig})\end{array}$} & $2.00 \pm 0.6$ & \multirow{5}{*}{2.28} & \multirow{5}{*}{$\begin{array}{l}0.04 \\
\text { (Sig) }\end{array}$} \\
\hline Primary school & $2.67 \pm 0.70$ & & & $2.33 \pm 0.70$ & & \\
\hline High school & $2.14 \pm 0.37$ & & & $1.86 \pm 0.69$ & & \\
\hline Higher secondary & $1.50 \pm 0.54$ & & & $1.83 \pm 0.75$ & & \\
\hline Graduate and above & $1.00 \pm 0.00$ & & & $1.00 \pm 0.00$ & & \\
\hline \multicolumn{7}{|c|}{ Occupation } \\
\hline Employed & $1.00 \pm 0.00$ & \multirow{3}{*}{15.41} & \multirow{3}{*}{$\begin{array}{c}<0.001 \\
(\mathrm{Sig})\end{array}$} & $1.25 \pm 0.50$ & \multirow{3}{*}{6.24} & \multirow{3}{*}{$\begin{array}{c}0.006 \\
\text { (Sig) }\end{array}$} \\
\hline Self employed & $1.40 \pm 0.54$ & & & $1.20 \pm 0.44$ & & \\
\hline House wife & $2.43 \pm 0.59$ & & & $2.19 \pm 0.75$ & & \\
\hline \multicolumn{7}{|c|}{ Parity } \\
\hline Primipara & $2.50 \pm 0.61$ & \multirow{3}{*}{13.30} & \multirow{3}{*}{$\begin{array}{c}<0.001 \\
(\mathrm{Sig})\end{array}$} & $2.22 \pm 0.73$ & \multirow{3}{*}{5.04} & \multirow{3}{*}{$\begin{array}{c}0.014 \\
\text { (Sig) }\end{array}$} \\
\hline Multipara & $1.50 \pm 0.52$ & & & $1.50 \pm 0.70$ & & \\
\hline Grand multipara & $1.00 \pm 0.00$ & & & $1.00 \pm 0.00$ & & \\
\hline \multicolumn{7}{|c|}{ Baby delivered at gestational week } \\
\hline$<36$ weeks (Preterm) & $1.00 \pm 0.00$ & \multirow{3}{*}{13.90} & \multirow{3}{*}{$\begin{array}{c}<0.001 \\
(\mathrm{Sig})\end{array}$} & $1.17 \pm 0.40$ & \multirow{3}{*}{4.36} & \multirow{3}{*}{$\begin{array}{c}0.023 \\
\text { (Sig) }\end{array}$} \\
\hline At $37-40$ weeks (Term) & $2.40 \pm 0.68$ & & & $2.15 \pm 0.81$ & & \\
\hline$>40$ weeks (Post term) & $2.00 \pm 0.00$ & & & $1.75 \pm 0.50$ & & \\
\hline \multicolumn{7}{|c|}{ Engorgement started from the day of delivery } \\
\hline $2^{\text {nd }}-3^{\text {rd }}$ day & $2.29 \pm 0.77$ & \multirow{3}{*}{3.58} & & $1.88 \pm .78$ & & \\
\hline $4^{\text {th }}-6^{\text {th }}$ day & $1.77 \pm 0.72$ & & $\begin{array}{l}0.06 \\
(N . S)\end{array}$ & $1.92 \pm .86$ & 0.018 & $\begin{array}{l}0.89 \\
(N . S)\end{array}$ \\
\hline$>7^{\text {th }}$ day & - & & & - & & \\
\hline & After deli & ery baby & atus & & & \\
\hline With mother & $1.74 \pm 0.653$ & ח 12 & $<0.001$ & $1.58 \pm 0.69$ & 1119 & 0.002 \\
\hline In NICU & $2.64 \pm 0.67$ & 12.90 & (Sig) & $2.45 \pm 0.68$ & 11.19 & (Sig) \\
\hline
\end{tabular}




\begin{tabular}{|c|c|c|c|c|c|c|}
\hline \multicolumn{7}{|c|}{ Previous knowledge about breast feeding practice } \\
\hline Yes & $1.12 \pm 0.35$ & \multirow{2}{*}{33.05} & \multirow{2}{*}{$\begin{array}{c}<0.001 \\
\text { (Sig) }\end{array}$} & $1.25 \pm 0.46$ & \multirow{2}{*}{9.15} & \multirow{2}{*}{$\begin{array}{c}0.005 \\
\text { (Sig) }\end{array}$} \\
\hline No & $2.41 \pm 0.59$ & & & $2.14 \pm 0.77$ & & \\
\hline \multicolumn{7}{|c|}{ Interval of breast feeding per day } \\
\hline Every 2 hourly & $1.67 \pm 0.50$ & \multirow{4}{*}{10.07} & \multirow{4}{*}{$\begin{array}{c}<0.001 \\
(\mathrm{Sig})\end{array}$} & $1.56 \pm 0.72$ & \multirow{4}{*}{2.96} & \multirow{4}{*}{$\begin{array}{l}0.05 \\
\text { (Sig) }\end{array}$} \\
\hline Every 3 hourly & $1.83 \pm 0.40$ & & & $2.00 \pm 0.63$ & & \\
\hline Every 4 hourly & $3.00 \pm 0.53$ & & & $2.50 \pm 0.75$ & & \\
\hline Demand feed & $1.71 \pm 0.75$ & & & $1.57 \pm 0.78$ & & \\
\hline \multicolumn{7}{|c|}{ Frequency of breast feeding per day } \\
\hline$<6$ times & $2.57 \pm 0.64$ & \multirow{3}{*}{16.75} & \multirow{3}{*}{$\begin{array}{c}<0.001 \\
\text { (Sig) }\end{array}$} & $2.14 \pm 0.86$ & \multirow{3}{*}{2.52} & \multirow{3}{*}{$\begin{array}{l}0.03 \\
\text { (Sig) }\end{array}$} \\
\hline 9-12 times & $1.62 \pm 0.61$ & & & $1.50 \pm 0.70$ & & \\
\hline$>12$ times & - & & & - & & \\
\hline \multicolumn{7}{|c|}{ Self-care method used for treatment of breast engorgement } \\
\hline Yes & $2.80 \pm 0.44$ & \multirow{2}{*}{6.17} & \multirow{2}{*}{$\begin{array}{l}0.09 \\
\text { (N.S) }\end{array}$} & $2.40 \pm 0.54$ & 2.44 & \multirow{2}{*}{$\begin{array}{c}0.12 \\
\text { (N.S) }\end{array}$} \\
\hline No & $2.2 \pm 0.75$ & & & $1.80 \pm 0.81$ & & \\
\hline \multicolumn{7}{|c|}{ Any top feed /supplement being given to neonate } \\
\hline Yes & $2.00 \pm 0.81$ & \multirow{2}{*}{0.032} & \multirow{2}{*}{$\begin{array}{l}0.85 \\
(N . S)\end{array}$} & $1.75 \pm 0.95$ & \multirow{2}{*}{0.156} & \multirow{2}{*}{$\begin{array}{c}0.69 \\
\text { (N.S) }\end{array}$} \\
\hline No & $2.08 \pm 0.79$ & & & $1.92 \pm 0.79$ & & \\
\hline
\end{tabular}

It is evident from Table 2, the mean post-test engorgement score of experimental and control group for right breast was shown $39 \%$ reduction of the engorgement score. This statistically significant. And for left breast was shown $40.50 \%$ reduction in engorgement score, this was found statistically significant as evident from $p<0.001$.

It is evident from table 3 , the mean post-test breast pain score of experimental and control group for right breast was shown $36 \%$ reduction of the pain score. This statistically significant and for left breast was shown 35\%reduction in pain score. This is statistically significant as evident from $\mathrm{p}<.001$
It is evident from table 4, Posttest breast engorgement score of experimental groups with selected demographic variables shows significant association with age in years, education, occupation, parity ,gestational weak, after delivery baby status, previous knowledge, interval of breast feeding/day and frequency of breast feeding / day $\mathrm{P}<0.05$.

Posttest breast engorgement score of experimental group is not found associated with Engorgement started from the day of delivery, self care method used for treatment of breast engorgement and any top feed/supplement being given to neonate.

Table 5.Presenting association between the posttest breast pain score of experimental group with selected demographic variables.by used ANOVA

\begin{tabular}{|c|c|c|c|c|c|c|c|}
\hline \multirow{3}{*}{ S. No. } & \multirow{3}{*}{ Type of variables } & \multicolumn{6}{|c|}{ Mean breast Pain Score } \\
\hline & & \multicolumn{3}{|c|}{ Rt breast } & \multicolumn{3}{|c|}{ Lt breast } \\
\hline & & mean $\pm S D$ & $\mathbf{F}$ & p-value & mean $\pm S D$ & $\mathbf{F}$ & p-value \\
\hline \multirow{4}{*}{1.} & \multicolumn{7}{|c|}{ Age } \\
\hline & 18-23years & $1.22 \pm 1.4$ & \multirow{3}{*}{2.76} & \multirow{3}{*}{$\begin{array}{c}.08 \\
\text { (N.S) }\end{array}$} & $1.11 \pm 1.3$ & \multirow{3}{*}{3.04} & \multirow{3}{*}{$\begin{array}{c}.06 \\
\text { (N.S) }\end{array}$} \\
\hline & 24-29 years & $2.50 \pm 1.6$ & & & $2.38 \pm 1.3$ & & \\
\hline & 30-35 years & $1.20 \pm .83$ & & & $1.40 \pm 1.1$ & & \\
\hline \multirow{4}{*}{2.} & \multicolumn{7}{|c|}{ Educational status } \\
\hline & No formal Education & $2.75 \pm 1.7$ & \multirow{3}{*}{.841} & \multirow{3}{*}{$\begin{array}{c}.51 \\
\text { (N.S) }\end{array}$} & $2.50 \pm 1.7$ & \multirow{3}{*}{1.07} & \multirow{3}{*}{$\begin{array}{c}.39 \\
\text { (N.S) }\end{array}$} \\
\hline & Primary school & $1.89 \pm 1.6$ & & & $1.67 \pm 1.1$ & & \\
\hline & High school & $2.14 \pm 1.9$ & & & $1.86 \pm 1.4$ & & \\
\hline
\end{tabular}




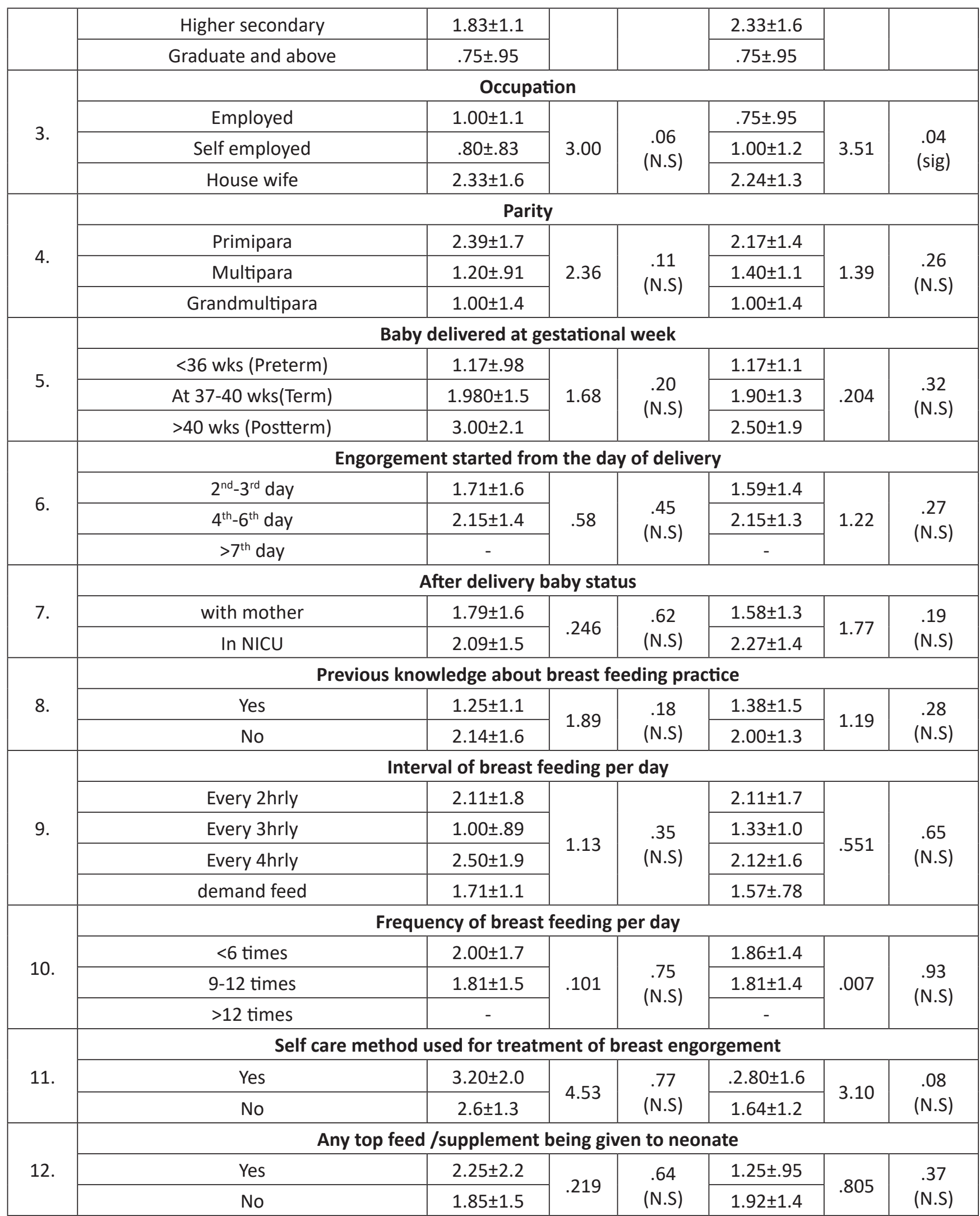

It is evident from table 5, Post test breast pain score of experimental group with selected demographic variables is found non significant $\mathrm{P}>0.05$.

\section{Discussion}

The present study revealed that the application of olive oil massage was very effective in breast engorgement and pain 
reduction among postnatal mothers who undergone LSCS with mean post-test engorgement score of experimental and control group for right breast was $2.07 \pm 0.79$ and $4.40 \pm 1.04$, there was $39 \%$ reduction of the engorgement score, this statistically significant as ' $\mathrm{t}$ ' value 8.726 is higher than the table value at 0.001 level of significance. And for left breast was $1.90 \pm 0.80$ and $4.33 \pm 1.21$, there was $40.50 \%$ reduction in engorgement score, this is statistically significant as ' $\mathrm{t}$ ' value 9.229 is higher than the table value at 0.001 level of significance. The mean post-test breast pain score of experimental and control group for right breast was $1.90 \pm 1.58$ and $5.53 \pm 1.74$ which shows $36 \%$ reduction of the pain score, this statistically significant as ' $t$ ' value 8.465 is greater than the table value at 0.000 level of significance and for left breast was $1.83 \pm 1.39$ and $5.33 \pm 1.94$, there was $35 \%$ reduction in pain score, this is statistically significant as ' $t$ ' value 8.045 is higher than the table value at 0.000 level of significance. The finding of this study was in conformity to another study conducted by Ms. P. Krishnaveni. ${ }^{4}$ It was a quasi experimental study to assess the effectiveness of breast massage on reduction of breast engorgement and pain among mother undergone caesarean section admitted in Balaji and Nallamuthusamy hospital at M.G.R Medical University, Tamilnadu. ${ }^{4}$ Purposive sampling used to select 60 mothers, 30 for experimental group and 30 for control group. The result of the study concluded that the breast massage is effective in reduction of breast engorgement and pain among mother undergone cesarean section there was a significant difference between mean pretest posttest level of breast engorgement among mother undergone cesarean section in experimental group ( $t=5.76, p<0.05)$. The present study was also supported by the findings of Jeongsug $C$ et al. ${ }^{5}$ Conducted a study to test the effect of oketani breast massage in the reduction of breast pain, the breast milk $\mathrm{pH}$ of mothers and the sucking speed of the neonates. The application of oketani massage by oketani massage therapist was the experimental treatment. The collected data were analysed using a $\mathrm{x}^{2}$-test and t-test with the SPSSWIN12.0 program. The result of the study concluded that Breast pain $(t=8.384, p<.001)$ was significantly relieved, and breast milk $\mathrm{Ph}(\mathrm{T}=4.793, \mathrm{P}<.001)$ was significantly increased in the experimental group compared to the control group. The sucking speed of neonate in experimental group was significantly increased compared to the control group $(t=9.920, p<.001)$. These finding indicate that oketani breast massage is effective in relieving breast pain and increasing breast milk pH as well as the sucking speed of neonates.

\section{Conclusion}

The pretest score of breast engorgement and pain was measured by six point engorgement scale and numerical pain rating scale, It was found slightly higher in experimental group as compared to control group, also application of olive oil massage on breast engorgement and pain was found to be effective in reducing breast engorgement scoreless up to $79 \%$ for both right and left breast and breast pain scoreless up to $71 \%$ for both right and left breast. Thus we can conclude that olive oil massage on engorged and painful breast helpful in reducing breast engorgement and pain intensity and it is effective, non-pharmacologic, accessible, cost-effective and noninvasive technique.

\section{Recommendation for Further Study}

- The olive oil massage is cost effective, safe, noninvasive, non-pharmacologic and effective method and can be utilized effectively without any side effects.

- A similar study can be conducted without using control group.

- A comparative study can be done by comparing olive oil massage with other intervention.

- A similar study can be done on postnatal mothers with normal vaginal delivery with using control group.

\section{Acknowledgment}

We take this opportunity to express our gratitude towards Ex. Capt. Geeta Parwanda, principal Subharti Nursing College, Meerut for providing all facilities and support to conduct this study smoothly. A word of appreciation goes to Dr. Mrs. Sudha Rastogi, CMS Dist. Women Hospital, and Meerut for giving me permission to conduct my study in the hospital. My immense thanks go to Mrs. TajNisha Banu, Reader, Head of the Dept. of Obstetrics and Gynecological Nursing, P.D.M Subharti Nursing college for her deep involvement, scholarly suggestions, timely corrections, support and motivations. I specially thanks with the greatest pleasure to my Father, Mother, Brother and sister for all their Love, Encouragement which gave confidence to achieve the goal.

\section{Conflict of Interest: None}

\section{References}

1. Breast engorgement. Available from: http:// en.m.wikipedia.org>wiki. 2015.

2. Moon JL, Humenick SS. Breast engorgement: contributing variables and variables amenable to nursing intervention. JOGNN 1989; 18(4): 309-315. [PubMed/ Google Scholar].

3. Schubiger G, Schwarz U, Tonz O. UNICEF/WHO babyfriendly hospital initiative: does the use of bottles and pacifiers in the neonatal nursery prevent successful breastfeeding? European Journal of Paediatrics 1997; 156(11): 874-877. [PubMed/ Google Scholar].

4. P. Krishnaveni. (2014). Effectiveness of breast massage on reduction of breast engorgement, Available from: http://repository-tnmgrmu.ac.in/10131/ 1/3003136krishnaveni.pdf.

5. Jeongsug C, Ahn HY et al. Effect of oketani breast massage in the reduction of breast pain, the breast milk ph of mothers and the suckling speed of the neonates. Korean Journal of Woman Health Nurse2012; 18(2): 149-158. 